\title{
Análisis crítico de un artículo: La terapia de reemplazo con la combinación de T3 con T4 no sería mejor que T4 sola en pacientes hipotiroideos
}

\author{
Rafael Téllez $\mathbf{T}^{1}$.
}

Critical appraisal: Combined T3 and T4 replacement therapy is not better than replacement with $\mathrm{T4}$ alone Saravanan P, Simmons DJ, Greenwood R, Peters TJ, Dayan CM. Partial substitution of thyroxine with tri-iodothyronine in patients on thyroxine replacement therapy: results of a large community-based randomized controlled trial. J Clin Endocrinol Metab 2005; 90: 805-12. studies may have been underpowered to detect differences in psychological well-being specifically related to thyroxine replacement. We conducted a large, double-blind, randomized controlled trial of partial substitution of 50 microg of T4 by 10 microg of T3 (T3) vs placebo (T4 alone - 50 microg of T4 replaced) in 697 hypothyroid patients. Thyroid function showed a rise in the TSH (132\%), a fall in Free T4 $(35 \%, \mathrm{P} \varangle 0.001)$ and unchanged basal Free T3 levels ( $\mathrm{P}=0.92)$. At 3 months there was a large (39\%) «placebo effect» improvement in «psychiatric caseness» defined by the General Health Questionnaire 12 score (GHQ 12) in the control group compared with baseline and this was sustained at 12 months. Differences vs the intervention (T3) group were more modest with improvements in GHQ caseness (OR - 0.61; 95\% CI: $0.42,0.90 ; \mathrm{P}=0.01$ ) and HADS anxiety scores at 3 months ( $\mathrm{P}$ $\varangle 0.03$ ) but not GHQ Likert scores, HADS depression, thyroid symptoms or visual analog scales of mood and the initial differences were lost at 12 months. These results may be consistent with a subgroup of patients showing transient improvement following partial substitution with T3 but do not provide conclusive evidence of specific benefit from partial substitution of T4 by T3 in patients on thyroxine replacement. They also emphasize the large and sustained placebo effects that can follow changes in thyroid hormone administration.

\footnotetext{
${ }^{1}$ Servicio de Medicina y Endocrinología, Hospital Sótero del Río. Santiago de Chile. Revisado por Unidad de Medicina Basa-
} da en Evidencia UC (UMBE). 


\section{CONCLUSIÓN DE LOS REVISORES:}

Aunque este trabajo reclutó un número importante de pacientes, no logró demostrar que el tratamiento de reemplazo tiroideo con la combinación de T4 con T3 sea mejor que T4 sola. Sin embargo, no descarta la posibilidad que algunos pacientes obtengan mejor alivio sintomático con esta asociación.

\section{PREGUNTA CĹNICA:}

¿El tratamiento con una combinación de T4 con dosis pequeñas de T3 es mejor que T4 sola para el control de los síntomas del hipotiroidismo?

\section{Contexto:}

El tratamiento del hipotiroidismo consiste en la administración de dosis de reemplazo de tiroxina (T4) solamente, a pesar de que la glándula tiroides secreta también triyodotimnina (T3), que es el ligando del receptor de hormona tiroidea. La administración de T3 tiene desventajas respecto del tratamiento con T4, debido a las grandes fluctuaciones de los niveles séricos de T3 que se producen. Aun dosis relativamente pequeñas de T3 pueden originar concentraciones séricas supranormales durante varias horas ${ }^{1}$. Sin embargo, cuando con la administración solo de T4 se logran niveles de TSH dentro del rango de referencia, las concentraciones de T3 libre circulantes están alrededor del límite inferior del rango normal y algunos pacientes siguen teniendo síntomas. Un estudio publicado en 1999, que involucró un número pequeño de pacientes, mostró mejoría significativa del humor en aquellos sujetos a los que se les sustituyó $50 \mathrm{mcg}$ de su dosis habitual de T4 por 12,5 mcg de T3, pero no hubo efecto sobre funciones cognitivas ${ }^{2}$. Trabajos posteriores, randomizados, pero también con números relativamente pequeños de pacientes, no encontraron beneficios.

\section{Características Del ESTUdio:}

Tipo de estudio: randomizado. Pacientes: adultos con hipotiroidismo, controlados en atención primaria, en tratamiento con dosis iguales o mayores de $100 \mathrm{mcg} /$ día de T4. Intervención: combinación de T4 con T3: a la dosis habitual de T4 se le quitó 50 mcg y se sustituyó con una tableta de $10 \mathrm{mcg}$ de T3. Comparación: T4 en dosis habitual menos 50 mcg, que se dan en una tableta aparte idéntica a la tableta de T3 (placebo»). Outcomes: Primarios: cambios en los puntajes del General Health Questionnaire (GHQ-12) a los 3 meses de tratamiento, respecto de la basal; las respuestas a este cuestionario se analizaron por un método Likert (como variables continuas, a menor puntaje mayor bienestar psicológico) y uno categórico (GHQ, con un puntaje umbral = 3 para catalogar probabilidad de morbilidad psiquiátrica). Secundarios: niveles de TSH, hormonas tiroideas, colesterol y otros parámetros bioquímicos a los 3 y $12 \mathrm{~m}$ de tratamiento; peso, presión arterial, porcentaje de grasa corporal, varios cuestionarios para evaluar bienestar psicológico y otro cuestionario acerca de síntomas específicos de enfermedad tiroidea a los 3 y 12 $\mathrm{m}$ de tratamiento. Seguimiento: $94,7 \%$ de los pacientes tenía datos para evaluación de outcomes a los 3 meses y $87,7 \%$ a los 12 meses. No se consignó cuántos sujetos se perdieron en cada grupo. 
VALIDEZ:

\begin{tabular}{|ll|}
\hline 1. Randomizado: & Sí \\
2. Ocultamiento de la secuencia de asignación: & No \\
3. Seguimiento: & $94,7 \%$ a los 3 meses \\
4. Análisis con intención de tratar: & Sí \\
5. Fueron ciegos a la intervención & \\
Pacientes: & Sí \\
Tratantes: & Sí \\
Recolectores de datos: & No informado \\
Adjudicadores de eventos: & No informado \\
Analistas de datos: & No informado \\
6. Grupos similares respecto a variables pronósticas conocidas: & Sí
\end{tabular}

RESULTADOS

\begin{tabular}{|lllcc|}
\hline Evento evaluado & $\begin{array}{l}\text { Tasa de } \\
\text { eventos Grupo } \\
\text { Intervención }\end{array}$ & $\begin{array}{l}\text { Tasa de } \\
\text { eventos Grupo } \\
\text { Control }^{*}\end{array}$ & $\begin{array}{c}\text { ARR** } \\
(95 \% \text { IC) }\end{array}$ & $\begin{array}{c}\text { NNT } \\
(95 \% \text { IC) }\end{array}$ \\
\hline GHQ Likert & 10,67 & 11,13 & $\begin{array}{c}-0,46 \\
(-1,12-0,26) \\
0,074\end{array}$ & - - \\
GHQ categórico & $(2,75)$ & $(2,35)$ & $26,6 \%$ & 14 \\
& $19,2 \%$ & & $(0,012-0,136)$ & $(7-84)$ \\
\hline
\end{tabular}

${ }^{*}$ En el caso de GHQ Likert, puntaje a los 3 meses de tratamiento; entre paréntesis, diferencia con el puntaje basal. ${ }^{* *} \mathrm{ARR}=$ Reducción de riesgo absoluto; en el caso de GHQ Likert (parámetro lineal) es la diferencia de beneficio entre los grupos. IC= Intervalo de confianza. NNT= Número necesario para tratar.

\section{COMENTARIOS Y APLICACIÓN PRÁCTICA:}

* Aunque reunió un número considerablemente mayor de pacientes que ensayos anteriores, este estudio no logró demostrar claramente un beneficio de la adición de T3 a la terapia de reemplazo tiroideo. Sólo hubo una leve pero significativa superioridad de la combinación T4/T3 a los 3 meses de tratamiento en los puntajes del GHQ-12 (expresado como variable discreta de probabilidad de morbilidad psiquiátrica; ARR= $7,4 \%$ ) y en los puntajes del cuestionario HADS de ansiedad (outcome secundario, $\mathrm{ARR}=4,5 \%)$.

* A los 3 meses hubo mejoría significativa del bienestar psicológico medido por un descenso de similar magnitud en los puntajes GHQ Likert en el grupo tratado con T4 y $\mathrm{T} 3$ y en el grupo control (2,75 y 2,35, respectivamente). La mejoría observada en el grupo control, que mantuvo la misma dosis total de T4 que antes de entrar al ensayo, podría atribuirse a efecto placebo. Como la diferencia en la respuesta entre los dos grupos es pequeña $(0,46)$ y con un intervalo de confianza $95 \%$ de $-1,12$ a 0,26, es difícil atribuirla a efecto terapéutico de T3. 
* No se informó si los adjudicadores de eventos fueron ciegos. Si no fue así, también pudo haber un sesgo en la estimación de la magnitud del efecto de T3.

* Con todo, es posible que algunos pacientes hipotiroideos necesiten recibir T3 como parte de su tratamiento para lograr alivio sintomático completo, impresión reforzada por trabajos en animales, en que el tratamiento sólo con tiroxina, aun cuando logre mantener eutiroidea a la hipófisis, deja tejidos en estado de hipotiroidismo ${ }^{3}$. Este ensayo clínico no logró aclarar la duda, pero sugirió que al menos un subgrupo de pacientes puede sentirse mejor con la combinación T4/T3. El número de sujetos randomizados fue insuficiente para demostrarlo, por la magnitud del efecto placebo observado al modificar la forma de administración de hormona tiroidea. Cuando se diseñen futuros ensayos deberá tenerse presente este efecto.

* Falta identificar qué enfermos se podrían beneficiar con la combinación T4/T3, cuál es la dosis y forma de administración de T3 más adecuada, qué marcadores de acción tiroidea a nivel tisular servirían para controlar el tratamiento (además de TSH) y qué efectos secundarios a largo plazo puede tener este tratamiento. Por el momento, el tratamiento estándar para el hipotiroidismo sigue siendo T4 sola.

\section{REFERENCIAS}

1. WeRNER \& IngBar's. The Thyroid: A fundamental and Clinical Text, Eighth Edition, edited by Lewis E. Braverman and Robert D. Utiger. Lippincott Williams \& Wilkins, Philadelphia, 2000.

2. Bunevicius R, Kazanavicius G, Zalinkevicius R, Prange Jr AJ. Effects of thyroxine as compared with thyroxine plus triiodothyronine in patients with hypothyroidism. NEngl J Med 1999; 340: 424-9.

3. Escobar-Morreale HF, Obregon MJ, Escobar DR et al. Replacement therapy for hypothyroidism with thyroxine alone does not ensure euthyroidism in all tissues, as studied in thyroidectomized rats. J Clin Invest 1995; 96: 2828-38. 